Pacific Journal of Mathematic 


\title{
TWO EXISTENCE THEOREMS FOR SYSTEMS OF LINEAR INEQUALITIES
}

\author{
Leonard M. Blumenthal
}

1. Introduction. In a previous paper [1], the writer initiated the development of the theory of linear inequalities by means of metric methods. This program is continued in the present note to obtain existence theorems for the solutions of two types of (finite) homogeneous systems of inequalities; existence criteria for such systems, different from those established in this paper, are given in the fundamental work of Theodore Motzkin [4]; see also [3].

If $A$ denotes an $m \times n$ matrix of real elements, and $x$ a column matrix of $n$ indeterminates, then the matrix $A x$ gives rise to the two systems of $m$ homogeneous linear inequalities in $n$ unknowns,

$$
A x \geq 0
$$

and

$$
A x \geqq 0,
$$

where the notation $\geq 0$ is interpreted to demand that at least one of the left members in ( 1 ) be positive. In this note necessary and sufficient conditions are found in order that these systems have a solution, which is nontrivial in the case of system (2). These conditions are expressed in terms of the signs of certain minors of the symmetric positive semi-definite matrix of order $m$ formed upon multiplying the matrix $A$ by its transpose $A^{T}$. They follow easily from a lemma concerning the distribution of $n+2$ points of the convexly metrized unit $n$-sphere $S_{n}$; this lemma is stated without proof in [2].

2. The Lemma. Let $p_{0}, p_{1}, \cdots, p_{n+1}$ be $n+2$ points of the $S_{n}$ and denote the geodesic distance of $p_{i}, p_{j}$ by $p_{i} p_{j}$; that is, $p_{i} p_{j}$ is the length of a shorter great circle arc that joins $p_{i}$ and $p_{j}$. Denoting the determinant

$$
\left|\cos p_{i} p_{j}\right| \quad(i, j=0,1, \cdots, n+1)
$$

Received January 22, 1952. The preparation of this paper was sponsored (in part) by the Office of Scientific Research, USAF. Presented to the American Mathematical Society, February, 1952.

Pacific J. Math. 3 (1953), 523-530 
by

$$
\Delta\left(p_{0}, p_{1}, \cdots, p_{n+1}\right)
$$

we recall the well-known result that

$$
\Delta\left(p_{0}, p_{1}, \cdots, p_{n+1}\right)=0
$$

while each principal minor of the determinant is nonnegative. If, moreover, a principal minor satisfies

$$
\Delta\left(p_{i_{0}}, p_{i_{1}}, \cdots, p_{i_{k}}\right) \neq 0
$$

then the points $p_{i_{0}}, p_{i_{1}}, \cdots, p_{i_{k}}$ are contained irreducibly in a $k$-dimensional (great) hypersphere $S_{k}$, and conversely. Clearly each $(m+1)$-tuple of such a set of $k+1$ points is contained irreducibly in an $S_{m}$.

LEMMA. If

$$
p_{0}, p_{1}, \cdots, p_{n}, p_{n+1} \in S_{n},
$$

with

$$
\Delta\left(p_{0}, p_{1}, \cdots, p_{n}\right) \neq 0
$$

then $(i)$ the points $p_{0}, p_{1}, \cdots, p_{n-1}$ determine uniquely an $(n-1)$-dimensional great hypersphere $S_{n-1}$, and (ii) the points $p_{n}, p_{n+1}$ lie on the same or on opposite sides of the hypersphere $S_{n-1}$ if and only if the cofactor $\left[\cos p_{n} p_{n+1}\right]$ of the element $\cos p_{n} p_{n+1}$ in $\Delta\left(p_{0}, p_{1}, \cdots, p_{n+1}\right)$ be negative or positive, respectively.

Proof. Since $\Delta\left(p_{0}, p_{1}, \cdots, p_{n}\right) \neq 0$ (and consequently is positive), the points $p_{0}, p_{1}, \cdots, p_{n}$ are irreducibly contained in $S_{n}$, and so $p_{0}, p_{1}, \cdots, p_{n-1}$ are irreducibly contained in a great hypersphere $S_{n-1}\left(p_{0}, p_{1}, \ldots, p_{n-1}\right)$ which they determine uniquely.

Let $s$ be any element of $S_{n}, s \neq p_{0}, p_{1}, \ldots, p_{n}$. Now

$$
\begin{aligned}
\Delta\left(p_{0}, p_{1}, \cdots, p_{n-1}\right) \Delta\left(p_{0}, p_{1}, \cdots, p_{n}, s\right) \\
\quad=\Delta\left(p_{0}, p_{1}, \cdots, p_{n}\right) \Delta\left(p_{0}, p_{1}, \cdots, p_{n-1}, s\right)-\left[\cos p_{n} s\right]^{2},
\end{aligned}
$$

and the vanishing of $\Delta\left(p_{0}, p_{1}, \cdots, p_{n}, s\right)$, together with the nonvanishing of $\Delta\left(p_{0}, p_{1}, \cdots, p_{n}\right)$, implies that $\left[\cos p_{n} s\right]=0$ if and only if 


$$
\Delta\left(p_{0}, p_{1}, \cdots, p_{n-1}, s\right)=0
$$

that is, if and only if $s \in S_{n-1}\left(p_{0}, p_{1}, \cdots, p_{n-1}\right)$.

It follows at once that if $p, q$ are any elements of $S_{n}$ which are on the same side of $S_{n-1}\left(p_{0}, p_{1}, \cdots, p_{n-1}\right)$, then

$$
\operatorname{sgn}\left[\cos p_{n} p\right]=\operatorname{sgn}\left[\cos p_{n} q\right],
$$

where $\left[\cos p_{n} p\right],\left[\cos p_{n} q\right]$ are cofactors of the indicated elements in the determinants $\Delta\left(p_{0}, p_{1}, \cdots, p_{n}, p\right), \Delta\left(p_{0}, p_{1}, \cdots, p_{n}, q\right)$, respectively. For in the contrary case, the continuous function $\left[\cos p_{n} s\right]$ changes sign for $s=p$ and $s=q$, and consequently it vanishes for some point of the geodesic (shorter) arc joining $p$ and $q$. But by the above, this point belongs to $S_{n-1}\left(p_{0}, p_{1}, \cdots, p_{n}\right)$, and so $p, q$ are on opposite sides of this great hypersphere, contrary to assumption.

If, therefore, $p_{n}$ and $p_{n+1}$ are on the same side of $S_{n-1}\left(p_{0}, p_{1}, \cdots, p_{n-1}\right)$, then

$$
\operatorname{sgn}\left[\cos p_{n} p_{n+1}\right]=\operatorname{sgn}\left[\cos p_{n} p_{n}\right]=-\operatorname{sgn} \Delta\left(p_{0}, p_{1}, \cdots, p_{n}\right),
$$

and consequently $\left[\cos p_{n} p_{n+1}\right]<0$.

Suppose, now, that $p_{n}$ and $p_{n+1}$ are on opposite sides of

$$
S_{n-1}\left(p_{0}, p_{1}, \cdots, p_{n-1}\right)
$$

and denote the reflection of $p_{n}$ in this hypersphere by $p_{n}{ }^{*}$. Then $p_{n}{ }^{*}, p_{n+1}$ are on the same side of the hypersphere, and so

$$
\operatorname{sgn}\left[\cos p_{n} p_{n+1}\right]=\operatorname{sgn}\left[\cos p_{n} p_{n}^{*}\right]
$$

From the vanishing of $\Delta\left(p_{0}, p_{1}, \cdots, p_{n}, p_{n}{ }^{*}\right)$ and the relations

$$
p_{i} p_{n}{ }^{*}=p_{i} p_{n} \quad(i=0,1, \cdots, n-1),
$$

which follow from the definition of $p_{n}^{*}$, (1) yields

$$
\left[\cos p_{n} p_{n}^{*}\right]= \pm \Delta\left(p_{0}, p_{1}, \cdots, p_{n}\right) \text {. }
$$

To determine the sign, we have, first, 


$$
\left[\cos p_{n} p_{n}^{*}\right]=-\left|\begin{array}{cccc}
1 & \cos p_{0} p_{1} & \cdots & \cos p_{0} p_{n} \\
\cos p_{0} p_{1} & 1 & \cdots & \cos p_{1} p_{n} \\
\cdot & \cdots & \cdots & \cdots \\
\cos p_{0} p_{n-1} & \cos p_{1} p_{n-1} & \cdots & 1 \\
\cos p_{0} p_{n}^{*} & \cos p_{1} p_{n}^{*} & \cdots & \cos p_{n-1} p_{n} \\
\cos p_{n} p_{n}^{*}
\end{array}\right| .
$$

Taking account of the relations $p_{i} p_{n}{ }^{*}=p_{i} p_{n}(i=0,1, \cdots, n-1)$, and writing the determinant as the sum of two determinants whose last rows are $\cos p_{0} p_{n}$, $\cos p_{1} p_{n}, \cdots, \cos p_{n-1} p_{n}, 1$ and $0,0, \ldots, 0, \cos p_{n} p_{n} *-1$, respectively, we easily obtain

$$
\begin{aligned}
& {\left[\cos p_{n} p_{n}^{*}\right]} \\
& \quad=-\Delta\left(p_{0}, p_{1}, \cdots, p_{n}\right)+\left(1-\cos p_{n} p_{n}^{*}\right) \Delta\left(p_{0}, p_{1}, \cdots, p_{n-1}\right) .
\end{aligned}
$$

Then clearly

$$
\left[\cos p_{n} p_{n}^{*}\right]=\Delta\left(p_{0}, p_{1}, \cdots, p_{n}\right)>0
$$

for if the negative sign were valid, substitution in (2) would give

$$
\left(1-\cos p_{n} p_{n}^{*}\right) \Delta\left(p_{0}, p_{1}, \cdots, p_{n-1}\right)=0
$$

But

$$
\Delta\left(p_{0}, p_{1}, \cdots, p_{n-1}\right) \neq 0
$$

because $p_{0}, p_{1}, \cdots, p_{n-1}$ are irreducibly contained in $S_{n-1}$, while, since $p_{0}$, $p_{1}, \cdots, p_{n-1}, p_{n}$ are irreducibly contained in $S_{n}, p_{n} \notin S_{n-1}\left(p_{0}, p_{1}, \ldots, p_{n-1}\right)$, and so $p_{n}$ is distinct from its reflection $p_{n}{ }^{*}$ in that hypersphere; that is,

$$
1-\cos p_{n} p_{n}^{*} \neq 0
$$

Hence if $p_{n}, p_{n+1}$ are on opposite sides of $S_{n-1}\left(p_{0}, p_{1}, \cdots, p_{n-1}\right)$, then $\left[\cos p_{n} p_{n+1}\right]>0$. To complete the proof, it suffices to observe that if

$$
\left[\cos p_{n} p_{n+1}\right] \neq 0
$$

then $p_{n+1} \notin S_{n-1}\left(p_{0}, p_{1}, \cdots, p_{n-1}\right)$. This is evident upon substituting $p_{n+1}$ for $s$ in (1). 
COROLLARY. Let $p_{0}, p_{1}, \cdots, p_{n+1}$ be pairwise distinct points of $S_{n}$, no $n+1$ of which are in a great hypersphere. If $\epsilon_{i j}=1$ or -1 according as $p_{i}$ and $p_{j}$ are on opposite sides or on the same side, respectively, of the great hypersphere $S_{n-1}\left(p_{0}, \cdots, p_{i-1}, p_{i+1}, \cdots, p_{j-1}, p_{j+1}, \cdots, p_{n+1}\right)$

$$
(i, j=0,1, \cdots, n+1 ; i \neq j)
$$

and $\epsilon_{i i}=1(i=0,1, \cdots, n+1)$, then the matrix $\left(\epsilon_{i j}\right)(i, j=0,1, \cdots, n+1)$ has rank 1 .

REMARK. In a manner similar to that employed above, companion theorems that characterize in a purely metric way the sides of hyperplanes in $n$-dimensional euclidean and hyperbolic spaces that are determined by a given set of $n$ points may be obtained. We state the euclidean theorem, which may be exploited to obtain existence theorems for systems of linear inequalities in much the same way as the lemma just proved will be used in the next section.

THEOREM 1. Let $p_{0}, p_{1}, \cdots, p_{n+1}$ be $n+2$ points of euclidean $n-$ space $E_{n}$ with $p_{0}, p_{1}, \cdots, p_{n}$ irreducibly contained in $E_{n}$. Then $p_{0}, p_{1}, \cdots, p_{n-1}$ determine a unique hyperplane $E_{n-1}$, and $p_{n}, p_{n+1}$ are on the same side or on opposite sides of this hyperplane if and only if

$$
\operatorname{sgn}\left[p_{n} p_{n+1}^{2}\right]=(-1)^{n} \text { or }(-1)^{n+1} \text {, }
$$

respectively, where $\left[p_{n} p_{n+1}^{2}\right]$ denotes the cofactor of $p_{n} p_{n+1}^{2}$ in the determinant

$$
D\left(p_{0}, p_{1}, \cdots, p_{n+1}\right)=\left|\begin{array}{ccccc}
0 & 1 & 1 & \cdots & 1 \\
1 & 0 & p_{0} p_{1}^{2} & \cdots & p_{0} p_{n+1}^{2} \\
1 & p_{0} p_{1}^{2} & 0 & \cdots & p_{1} p_{n+1}^{2} \\
\cdots & \cdot & \cdots & \cdots & \cdot \\
1 & p_{0} p_{n+1}^{2} & p_{1} p_{n+1}^{2} & \cdots & 0
\end{array}\right|
$$

We observe, moreover, that for $p_{0}, p_{1}, \cdots, p_{n}$ irreducibly contained in $E_{n}$ it may be shown that

$$
\begin{aligned}
& {\left[p_{n-1} p_{n}^{2}\right]} \\
& \begin{aligned}
(-1)^{n-1} 2^{n-1}[(n-1) !]^{2} V\left(p_{0}, \cdots\right. & \left., p_{n-2}, p_{n-1}\right) V\left(p_{0}, p_{1}, \cdots, p_{n-2}, p_{n}\right) \\
& \quad \times \cos \Varangle\left(p_{0}, p_{1}, \ldots, p_{n-2}: p_{n-1}, p_{n}\right),
\end{aligned}
\end{aligned}
$$


where $\left[p_{n-1} p_{n}{ }^{2}\right]$ is the cofactor of $p_{n-1} p_{n}{ }^{2}$ in $D\left(p_{0}, p_{1}, \ldots, p_{n}\right), V$ is the volume of the $(n-1)$-dimensional simplex determined by the points indicated, and $\Varangle\left(p_{0}, p_{1}, \ldots, p_{n-2}: p_{n-1}, p_{n}\right)$ denotes the “dihedral” angle with $(n-2)$ dimensional edge $E_{n-2}\left(p_{0}, p_{1}, \ldots, p_{n-2}\right)$ of the simplex with vertices $p_{0}$, $p_{1}, \cdots, p_{n-1}, p_{n}$.

Hence $\left[p_{n-1}{p_{n}}^{2}\right]=0$ if and only if $\Varangle\left(p_{0}, p_{1}, \cdots, p_{n-2}: p_{n-1}, p_{n}\right)=\pi / 2$, and $\operatorname{sgn}\left[p_{n-1} p_{n}^{2}\right]=(-1)^{n-1}$ if and only if the dihedral angle is acute.

It is, perhaps, worth pointing out that The orem 1 yields a purely metric characterization of a nondegenerate simplex (interior and boundary) of $E_{n}$. For if $p_{0}, p_{1}, \cdots, p_{n}$ are the vertices of such a simplex, a point $p$ of $E_{n}$ evidently belongs to its interior or boundary if and only if $p$ and $p_{i}$ are not on opposite sides of the hyperplane $E_{n-1}\left(p_{0}, \ldots, p_{i-1}, p_{i+1}, \cdots, p_{n}\right)(i=0,1, \ldots, n)$; that is, according to the theorem, if and only if

$$
\operatorname{sgn}\left[p_{i} p^{2}\right]=(-1)^{n} \text { or } 0 \quad(i=0,1, \cdots, n),
$$

where $\left[p_{i} p^{2}\right]$ is the cofactor of $p_{i} p^{2}$ in the determinant $D\left(p_{0}, p_{1}, \cdots, p_{n}, p\right)$.

Since a point of $E_{n}$ is contained in the convex extension of a $k$-tuple of $E_{n}$ ( not of $E_{n-1}$ ) if and only if it belongs to the simplex determined by some $n+1$ points of the $k$-tuple, the above observation yields a metric characterization of such convex extensions.

3. The theorems. We are now in position to prove the two existence theorems.

THEOREM 2. Let $A x \geq 0$ be a system of $m$ linear inequalities in $n$ indeterminates with rank $r+1$, and let $B$ denote the determinant of the matrix $A A^{T}$. The system has a solution if and only if a shifting of rows and corresponding columns of A exists such that

( $i$ ) the upper left principal minor $M$ of $B$ of order $r+1$ does not vanish,

(ii) each minor of $B$ formed from $M$ by replacing its last row with that part of the $j$-th row of $B$ contained in the first $r+1$ columns $(j=r+2, r+3, \cdots, m)$ is positive or zero.

Proof. Each row of $A$ gives, after normalization, a point of the unit $n$-sphere $S_{n}$, and since the rank of $A$ is $r+1$ it follows that the $m$ "row points" are contained irreducibly in an $r$-dimensional hypersphere $S_{r}$ of the $S_{n}$. Denoting by $p_{i}$ the point corresponding to the $i$-th row of $A(i=1,2, \cdots, m)$ after a shifting of rows and columns of $A$ in conformity with the hypotheses has been carried out, we see that $p_{1}, p_{2}, \cdots, p_{r}, p_{r+1}$ lie irreducibly in $S_{r}$, and that $p_{1}, p_{2}, \cdots, p_{r}$ determine a unique $(r-1)$-dimensional great hypersphere $S_{r-1}\left(p_{1}, p_{2}, \cdots, p_{r}\right)$. 
Now the cofactor $\left[\cos p_{r+1} p_{j}\right]$ of the element $\cos p_{r+1} p_{j}$ in the vanishing determinant $\Delta\left(p_{1}, p_{2}, \cdots, p_{r+1}, p_{j}\right)(j=r+2, r+3, \cdots, m)$ has the sign opposite to that of the minor of that element which, in turn, has the same sign as the minor of $B$ described in hypothesis $(i i)$. Hence

$$
\left[\cos p_{r+1} p_{j}\right] \leqq 0 \quad(j=r+2, \cdots, m)
$$

and so, by the lemma, each of the points $p_{r+2}, p_{r+3}, \cdots, p_{m}$ lies on the same side of $S_{r-1}\left(p_{1}, p_{2}, \cdots, p_{r}\right)$ as $p_{r+1}$, or is contained in that hypersphere.

Hence the $m$ points are contained in a hemi- $S_{r}$ with at least one of the $m$ points, $p_{r+1}$, not on the $S_{r-1}$ forming its "rim". The center of this hemi- $S_{r}$ is evidently a solution of the system of inequalities, and so the conditions stated in the theorem are sufficient. We have, indeed, found that the $S_{r}$ itself contains a solution of the system.

To extablish the necessity, we remark that if the system has a solution in $S_{n}$, then it has a solution in the $S_{r}$ containing the $m$ points $p_{1}, p_{2}, \cdots, p_{m}$ irreducibly. For if $p \in S_{\dot{n}}$ which is a solution of the system, then $p$ is the center of a hemi- $S_{n}$ which contains $p_{1}, p_{2}, \cdots, p_{m}$ and has at least one of these points, say, $p_{m}$, in its interior. But if the $S_{r}$ has no solution of the system, the (spherical ) convex extension of the $m$-tuple is the whole $S_{r}$, which must be contained in the hemi- $S_{n}$ on which the $m$ points lie. But this is impossible since the point $p_{m}{ }^{*}$ diametral to $p_{m}$ lies in the $S_{r}$ but it clearly does not belong to the hemi- $S_{n}$.

If, therefore, the system has a solution, there is a point of the $S_{r}$ containing $p_{1}, p_{2}, \cdots, p_{m}$ which is the center of a hemi- $S_{r}$ that contains $p_{1}, p_{2}, \cdots, p_{m}$, with at least one of these points in its interior. It is easily seen that any such hemi- $S_{r}$ may be rotated so as to retain this property and have some $r$ of the $m$ points, say $p_{1}, p_{2}, \cdots, p_{r}$, on the $S_{r-1}$ forming its rim. If $p_{r+1}$ is in the interior of the hemi- $S_{r}$ so obtained, then clearly each of the remaining points is either on its rim or on the same side of the rim as $p_{r+1}$. Invoking, now, the lemma, we see that the conditions of the theorem are satisfied.

REMARK. The direction-cosines of the normal to the hyperplane $E_{r}$ determined by the points $p_{1}, p_{2}, \cdots, p_{r}$ (and the origin) give a solution of the system of inequalities; but since these numbers are found by evaluating determinants, a solution method based on the theorem is probably not suitable for computing machines.

THEOREM 3. Let $A x \geqq 0$ be a system of $m$ linear inequalities in $n$ indeterminates. The system possesses a nontrivial solution if and only if whenever the 
rank of $A$ equals $n$, a shifting of rows and corresponding columns of the determinant $B$ of $A A^{T}$ exists such that $(i)$ the $n$th order upper left principal minor $M$ of $B$ is not zero, while ( $i i)$ each $n$th order minor of $B$ obtained from $M$ by replacing the last row of $M$ with that part of the $j$-th row contained in the first $n$ columns of $B$ is positive or zero for $j=n+1, n+2, \cdots, m$.

Proof. The rank of $A$ is, of course, at most $n$; and if it is less than $n$ then the $m$ row points lie in an $E_{n-1}$ containing the origin, the coefficients of which annul all the members of the system of inequalities and hence form a solution.

If the rank of $A$ equals $n$ then the row points are not contained in any $E_{n-1}$ passing through the origin, and so the system has a nontrivial solution if and only if the system $A x \geq 0$ has a solution; that is (by virtue of Theorem 3 ), if and only if conditions $(i)$ and $(i i)$ are satisfied.

\section{REFERENCES}

1. L. M. Blumenthal, Metric methods in linear inequalities, Duke Math. J. 15 (1948), 955-966.

2. L. M. Blumenthal and B. E. Gillam, Distribution of points in n-space, Amer. Math. Monthly 50 (1943), 181-186.

3. W. B. Carver, Systems of linear inequalities, Ann. of Math. 23 (1921-22), 212-220.

4. T. S. Motzkin, Beiträge zur Theorie der linearen Ungleichungen, Dissertation, Basel, 1933 (Jerusalem, 1936).

National Bureau of Standards, Los Angeles, and

University of Missouri 


\section{PACIFIC JOURNAL OF MATHEMATICS}

\section{EDITORS}

\section{R. M. RoBINSON \\ University of California Berkeley 4, California}

\author{
*R. P. Dilmorth \\ Califomia Institute of Technology \\ Pasadena 4, California
}

E. F. BeckenbaCh, Managing Editor

University of California

Los Angeles 24, California

${ }^{*}$ During the absence of Herbert Busemann in 1952.

\section{ASSOCIATE EDITORS}

$\begin{array}{llll}\text { R. P. DILWORTH } & \text { P. R. HALMOS } & \text { BØRGE JESSEN } & \text { J. J. STOKER } \\ \text { HERBERT FEDERER } & \text { HEINZ HOPF } & \text { PAUL LÉVY } & \text { E. G. STRAUS } \\ \text { MARSHALL HALL } & \text { R. D. JAMES } & \text { GEORGE PÓLYA } & \text { KÖSAKU YOSIDA }\end{array}$

\section{SPONSORS}

UNIVERSITY OF BRITISH COLUMBIA

CALIFORNIA INSTITUTE OF TECHNOLOGY

UNIVERSITY OF CALIFORNIA, BERKELEY

UNIVERSITY OF CALIFORNIA, DAVIS

UNIVERSITY OF CALIFORNIA, LOS ANGELES

UNIVERSITY OF CALIFORNIA, SANTA BARBARA

OREGON STATE COLLEGE

UNIVERSITY OF OREGON

\author{
UNIVERSITY OF SOUTHERN CALIFORNIA \\ STANFORD UNIVERSITY \\ WASHINGTON STATE COLLEGE \\ UNIVERSITY OF WASHINGTON \\ AMERICAN MATHEMATICAL SOCIETY \\ NATIONAL BUREAU OF STANDARDS, \\ INSTITUTE FOR NUMERICAL ANALYSIS
}

Mathematical papers intended for publication in the Pacific Journal of Miathematics should be typewritten (double spaced), and the author should keep a complete copy. Manuscripts may be sent to any of the editors. All other communications to the editors should be addressed to the managing editor, E. F. Beckenbach, at the address given above.

Authors are entitled to receive 100 free reprints of their published papers and may obtain additional copies at cost.

The Pacific Journal of Mathematics is published quarterly, in March, June, September, and December, by the University of California, Berkeley 4, California. The price per volume (4 numbers) is \$8.00; single issues, $\$ 2.50$. Special price to individual faculty members of supporting institutions and to individual members of the American Mathematical Society: $\$ 4.00$ per volume; single issues, $\$ 1.25$.

Subscriptions, orders for back numbers, and changes of address should be sent to the publishers, University of California Press, Berkeley 4, California.

Printed at Ann Arbor, Michigan. Entered as second class matter at the Post Office, Berkeley, California.

\section{UNIVERSITY OF CALIFORNIA PRESS • BERKELEY AND LOS ANGELES}




\section{Pacific Journal of Mathematics}

\section{Vol. 2, No. $4 \quad$ April, 1952}

Shmuel Agmon, On the singularities of Taylor series with reciprocal coefficients .................................... 431

Richard Arens, A generalization of normed rings ............... 455

Iacopo Barsotti, Intersection theory for cycles of an algebraic variety . . . . 473

Leonard M. Blumenthal, Two existence theorems for systems of linear inequalities ..................................... 523

Frank Herbert Brownell, III, Translation invariant measure over separable Hilbert space and other translation spaces................. 531

J. W. S. Cassels, On a paper of Niven and Zuckerman ............... 555

Nelson Dunford, Spectral theory. II. Resolutions of the identity .......... 559

Eugene Lukacs and Otto Szász, On analytic characteristic functions ...... 615

W. A. Mersman, Evaluation of an integral occurring in servomechanism theory.......................................... 627

Lawrence Edward Payne and Alexander Weinstein, Capacity, virtual mass, and generalized symmetrization......................... 633

Choy-Tak Taam, The boundedness of the solutions of a differential equation in the complex domain ...................................... 643 